\title{
Frage nach dem entwicklungspolitischen Nutzen und der effizienten Verwendung öffentlicher Gelder
}

Dominik Egli

\section{(2) OpenEdition Journals}

Édition électronique

URL : http://journals.openedition.org/sjep/1432

ISSN : 1663-9677

\section{Éditeur}

Institut de hautes études internationales et du développement

\section{Édition imprimée}

Date de publication : 1 janvier 1994

Pagination : 286-290

ISSN : $1660-5926$

Référence électronique

Dominik Egli, « Frage nach dem entwicklungspolitischen Nutzen und der effizienten Verwendung öffentlicher Gelder », Schweizerisches Jahrbuch für Entwicklungspolitik [En ligne], 13| 1994, mis en ligne le 10 juin 2013, consulté le 07 septembre 2020. URL : http://journals.openedition.org/sjep/1432 


\section{Frage nach dem entwicklungspolitischen Nutzen und der effizienten Verwendung öffentlicher Gelder}

Dominik Egli

Im Zentrum von Kappels Analyse steht die Frage nach dem entwicklungspolitischen Nutzen der schweizerischen Entschuldungsfazilität. Kappel identifiziert zwei potentielle Quellen für durch Schuldenreduktion induzierte Besserstellung von überschuldeten Ländern. Erstens kann durch eine Schuldenreduktion der Zugang zum internationalen Kapitalmarkt wiederhergestellt werden, und zweitens soll eine Reduktion Reformen auslösen, die zu einer Einkommenserhöhung der begünstigten Länder führen sollen.

\section{Zugang zum internationalen Kapitalmarkt}

Eine Schuldenkrise kann definiert werden als ein Zustand, in dem das, was das Land aufgrund der abgeschlossenen Verträge zahlen müsste höher ist als das, was das Land effektiv in der Lage oder bereit ist zu bezahlen. Es ist naheliegend, dass in einer solchen Situation kein Gläubiger bereit ist, weitere Kredite in das Land fliessen zu lassen. Ebenso einleuchtend ist, dass sich die Lage ändert, wenn die Schuld durch Aktionen Dritter soweit reduziert wird, dass das Land weniger zahlen muss als es bereit ist. Dann nämlich werden Gläubiger bereit sein, die entstandene Lücke zwischen Zahlungsversprechen und Zahlungsbereitschaft durch Neukredite zu füllen. Insofern kann ein überschuldetes Land zweifelsohne von Schuldenrückkäufen profitieren. Offen bleibt, ob dies eine effiziente Verwendung von öffentlichen Geldern darstellt. Wenn die Schweiz aus Anlass ihres runden Geburtstages 400 Millionen Franken aufwenden will, um via Schuldrückkäufen ärmsten Ländern zu helfen, muss zuallererst die Frage gestellt werden, ob dieses Geld nicht durch anderweitige Aktionen mehr Nutzen stiften könnte. Bei Kappels erstem Argument besteht der Nutzen für das Land aus Neugeldzuflüssen, die durch Schuldreduktionen ausgelöst werden sollen. Anstatt den Umweg über Rückkäufe bestehender Schuld zu nehmen, könnten die 400 Millionen Franken auch direkt dem Land geschenkt werden. Die Frage ist, wie die Rückkaufaktion im Vergleich zu einem direkten Geschenk abschneidet. Betragen die Neuzuflüsse in die begünstigten Länder mehr oder weniger als 400 Millionen Franken ?

Das Problem sei an einem stark vereinfachten Beispiel dargestellt (1). Nehmen wir an, ein Land schulde einer ausländischen Bank einen Betrag von 100 mit unendlicher Laufzeit und Verzinsung von 10\% pro Jahr. Die Verzinsung entspreche derjenigen alternativer risikoloser Anlagen. Nun sei das Land nicht mehr in der Lage, die jährlichen Zahlungen von 10 zu leisten, und es erklärt der Bank, mehr als 5 pro Jahr sei auf alle Zeiten hin nicht möglich. Eine gutgesinnte Regierung überlegt sich nun, wie sie dem Land helfen könnte. Kappels Argument 
lautet in diesem Beispiel folgendermassen: Wenn die Regierung bei der Bank sagen wir $70 \%$ der Schuld zurückkauft, reduziert sich der Schuldendienst des Landes auf 3 pro Jahr. Da das Land bereit ist, 5 pro Jahr zu bezahlen, wird sich ein Gläubiger finden, der dem Land einen Neukredit in Höhe von 20 zu 10\% Verzinsung jährlich vergibt. Damit findet ein Mittelzufluss in das Land statt, wovon es profitiert. Welche Kosten stehen dieser Nutzensteigerung gegenüber? Wieviel muss die gutgesinnte Regierung der Bank bezahlen, damit diesé bereit ist, $70 \%$ der Schuld zu verkaufen? Die Bank nimmt ein Angebot nur an, wenn sie dabei zumindest nicht schlechter gestellt wird. Nach dem Rückkauf verbleibt ihr ein sicherer jährlicher Ertrag aus dem verbleibenden Anspruch gegenüber dem Land von 3. Sie wird also bereit sein, $70 \%$ ihres Anspruches zu verkaufen, wenn sie dafür einen Gegenwert von 2 pro Jahr erhält. Bei einem risikolosen Zinssatz von 10\% muss die Regierung der Bank für $70 \%$ ihres Anspruches also mindestens 20 bezahlen.

Das Land erhält somit durch die Aktion einen Neuzufluss von 20, die Regierung wendet genau diesen Betrag auf. Exakt denselben Effekt könnte die Regierung auch durch ein direktes Geschenk erzielen. Bis hierher habe ich von sämtlichen Durchführungskosten abstrahiert. Die Rückkaufaktion ist aber, insbesondere wenn mehrere Gläubiger betroffen sind, aufgrund der notwendigen Verhandlungen mit Sicherheit aufwendiger, sodass bei Miteinbezug der Transaktionskosten das Geschenk effizienter ist.

Nun werden Schuldtitel von internationalen Kreditbeziehungen in aller Regel auf einem Sekundärmarkt gehandelt. Bei Ländern, die in Zahlungsverzug sind, werden diese Titel mit einem Abschlag auf dem Nennwert notiert. Vordergründig scheint es verlockend zu sein, über den Markt billig Schulden armer Länder aufzukaufen und den betreffenden Ländern zu schenken. Bei einem Sekundärmarktpreis von beispielsweise $10 \%$ könnte man mit 100 Millionen Franken Schulden in Höhe von 1 Milliarde Franken aufkaufen und dem Land erlassen. Damit müsste doch der Nutzen die Höhe der aufgewendeten Mittel übersteigen. Nehmen wir für unser obiges Beispiel an, es gäbe nicht nur eine Gläubigerbank, sondern einhundert, von denen jede einen Anspruch in Höhe von 1 halten würde. Diese Ansprüche würden auf einem Markt gehandelt. Der Marktpreis für einen Titel beträgt 0,5 , da jemand, der einen Anteil im Nominalwert von 1 zu diesem Preis ersteht, sich eine Verzinsung von $10 \%$ garantiert, genau das, was er auf andere risikolose Anlagen auch erhalten würde. Wenn nun aber die Gläubigerbanken wissen, dass die Regierung die Titel, die sie aufkauft, vernichten wird, sind sie nicht mehr bereit, zum Preis von 0,5 zu verkaufen. Sie wissen nämlich, dass die vom Land zu erhaltenden Zahlungen in Zukunft auf weniger Gläubiger aufgeteilt werden. Die Rückkaufaktion senkt die Nominalschuld, sodass jeder verbleibende Gläubiger mehr erhält. Will die Regierung die Nominalschuld auf unter 50 senken, muss sie die Titel zum Nennwert kaufen. Ein Rückkauf von $70 \%$ der Titel würde dann 70 kosten, der Nutzen für das Land wäre unverändert. Hier wäre somit ein Geschenk effizienter.

Dass dieses Gedankenspiel nicht bar jeder Realitätsnähe ist, zeigt die sogenannte Bolivien-1 Aktion (Bulow und Rogoff 1988). 1988 führte Bolivien einen 
Rückkauf mit Mitteln von dritten Parteien durch. Die gegenüber den privaten Gläubigern ausstehende Schuld belief sich auf 670 Millionen US-Dollar, der Sekundärmarktpreis betrug 0,06 . Der Wert der ausstehenden Schuld betrug damit 40,2 Millionen \$. Die Drittparteien stellten 34 Millionen \$ zur Verfügung. Der Kaufpreis entsprach dem Sekundärmarktpreis nach der Aktion und betrug 0,11. Damit gelang es, die Schuld um 308 Millionen \$ zu kürzen. Der Wert der verbleibenden Schuld betrug 39,8 Millionen \$. Von den eingesetzten 34 Millionen $\$$ gingen somit 4001000 \$ an Bolivien, der Rest an die Gläubiger.

Als weiteres Beispiel ist die schweizerische Rückkaufaktion von Lieferantenschulden zu nennen. Für 59 Millionen SFr. wurden Selbstbehaltanteile schweizerischer Exporteure aus der ERG aufgekauft. Damit konnten für insgesamt 22 Länder die ausstehenden Handelskredite gegenüber schweizerischen Exporteuren um $95 \%$ gekürzt werden. Der durchschnittliche Preis, der bezahlt werden musste, belief sich auf 0,19 , der Sekundärmarktpreis privater Bankschulden auf 0,15. Damit konnten insgesamt Schuldtitel im Nominalwert von $\mathbf{3 1 0}$ Millionen Franken erworben werden (2). Der Wert der ausstehenden Schulden der schweizerischen Exporteure vor dem Rückkauf beläuft sich auf 46,5 Millionen Franken (0,15 mal 310). Dank der Bezahlung eines Preises von 0,19 flossen von den eingesetzten 59 Millionen Franken 12,5 Millionen direkt in die Kassen schweizerischer Unternehmen.

\section{Unterstützung von Reformprogrammen}

Hinter diesem Argument steckt die Annahme, dass überschuldete Länder an sich sinnvolle Reformen aufgrund ihrer Verschuldung nicht durchführen, weil zumindest ein Teil des Erfolges solcher Programme an die Gläubiger abfliessen würde. Dies ist der in der Literatur häufig genannte "Schulden-Laffer-Kurven-Effekt" (Sachs 1988, Froot 1989). Die für diesen Effekt notwendige und problematische Annahme ist diejenige des Anstiegs der Zahlungswilligkeit eines überschuldeten Landes bei steigendem Bruttosozialprodukt. Wie Kappel auch feststellt, besteht das dominierende Problem bei internationalen Kreditbeziehungen in der Zahlungsmoral der Schuldner. Es gibt auf internationaler Ebene kein Konkursverfahren, das die Schuldner diszipliniert. Die Annahme von zum Einkommen proportionaler Zahlungswilligkeit verwendet aber implizit die Annahme einer Institution, die die Zahlung eines bestimmten Anteiles des Einkommens an die Gläubiger erzwingt. Andernfalls müsste der Zusammenhang zwischen Einkommen und Strafpotential aufgezeigt werden. Eine Möglichkeit wäre, von der Annahme auszugehen, die Gläubiger könnten im Verzugsfall die Aussenhandelstätigkeit des Schuldners verteuern. Der Effekt bleibt hierbei zwar bestehen, ist aber geringer, da die Schuldner durch die Umstellung der Produktion reagieren können.

Zusätzlich sind hier ebenfalls die durch die Öffentlichkeit aufzuwendenden Kosten mit dem zu erzielenden Nutzen zu vergleichen. Der Laffer-Kurven-Effekt muss also stark genug sein, um die oben gezeigte Problematik zu dominieren. Empirische Arbeiten kommen zu wiedersprüchlichen Resultaten. Geschätzt wurde 
jeweilen der Einfluss der Schuldhöhe auf die Investitionstätigkeit. Die theoretische Argumentation ist dieselbe: Investitionen führen zu einem höheren BSP, was wiederum erhöhte Zahlungen an die Gläubiger zur Folge hat. Dies führt zu einer Verzerrung der Investitionsanreize. Reformprogramme können auch als Investitionen betrachtet werden.

Für die siebzehn höchstverschuldeten Länder schätzt Eaton (1990) den Einfluss der Höhe des Bruttosozialprodukts auf die Höhe der Rückzahlungen auf $1,1 \%$. Eine Erhöhung des Outputs um eine Einheit führt demzufolge zu einer Erhöhung der Zahlungen um 0,01 Einheiten. Dies ist eine "Grösse", die die Investitionstätigkeit kaum beeinflussen dürtte.

Cohen (1990) schätzte für 81 Länder den Einfluss von Bevölkerungswachstum, Inflation, des Export/Output-Verhältnisses, des Pro-Kopf-Einkommens und des Schuld/Export-Verhältnisses auf die Investitionen. Sein Resultat kann einen Einfluss der Schuld nicht stützen.

Borensztein (1990) schätzt für die Philippinen den Einfluss der Investitionsgüterpreise, der Grenzproduktivität des Kapitals, der erwarteten Realzinsen und verschiedener Schuldvariablen auf die Investitionstätigkeit. Die Hypothese der Neutralität der Schuld wird in dieser Studie verworfen.

Alle diese Schätzungen sind aus demselben Grund problematisch. Möglicherweise wurden sowohl der Rückgang der Investitionen wie auch die Schuldenkrise durch dieselben exogenen Schocks (Rohstoffpreisfall, Zinsanstieg, Rezession in den Industrieländern) ausgelöst. Ist dies der Fall, sind die erklärenden Variablen in den oben dargestellten Schätzungen endogen, die Resultate damit nicht verlässlich.

Savvides (1992) versucht das Problem durch ein simultanes Gleichungsmodell zu umgehen und kommt zum Resultat, dass Schuldbestände einen Einfluss auf die Investitionshöhe haben.

Warner (1992) versucht, das Problem auf andere Art zu umgehen. Aufgrund von Vergangenheitsdaten schätzt er die zu erwartende Reaktion der Investitionstätigkeit auf die vorgekommenen exogenen Schocks. Seine Hypothese lautet, dass die tatsächlichen Investitionen tiefer liegen müssen, damit ein Einfluss der Schuldenkrise nicht abgelehnt werden kann. Bei elf der dreizehn untersuchten Länder (Venezuela, Marokko, Equador, Mexiko, Kolumbien, Bolivien, Chile, Brasilien, Argentinien, Uruguay, Peru, die Philippinen und Nigeria) musste die Hypothese verworfen werden. Die beiden Ausnahmen sind Nigeria und Argentinien.

\section{Zusammenfassung}

Kappels Argumentation vermag einer Effizienzbetrachtung nicht standzuhalten. Wenn das Ziel des schweizerischen Geschenkes sein soll, den ärmsten Ländern zu helfen, ist meines Erachtens zuallererst die Frage zu stellen, wie man dies mit den vorhandenen Mitteln am besten macht. Werden hierbei vor allem Exporteure und Banken für ihre Fehler entschädigt, anstatt dass das Geld in die Länder fliesst, schiesst die Aktion am gesteckten Ziel vorbei. 


\section{Anmerkungen}

1. Für eine formale Darstellung siehe Egli 1993.

2. Durch die gleichzeitige Streichung der ERG-Forderung in Höhe von 850 Millionen Franken resultiert ein Schuldenerlass von 1,1 Milliarden Franken. Die gesamte Schuld als Erfolg der Faziliät abzuhaken, ist nicht gerechtfertigt. Implizit bezahlt der Steuerzahler den Preis für die erlassene ERG-Schuld.

\section{Literatur}

Borensztein Eduardo: Debt Overhang, Debt Reduction, and Investment: The Case of the Philippines. IMF Working Paper 1990

Bulow Jeremy, Kenneth Rogoff: The Buyback Boondoggle. Brookings Papers on Economic Acitvity 1988

Cohen Daniel: Slow Growth and large LDC Debt in the Eighties: An Update. CEPREMAP Paris 1990

Eaton Jonathan: Debt Relief and the International Enforcement of Loan Contracts. The Journal of Economic Perspectives 1990

Egli Dominik: Die schweizerische Entschuldungsfazilität - ein Flop? Diskussionspapier No 11 des Nationalen Forschungsprogrammes 28. Schweizerischer Nationalfonds Bern, 1993 Froot Kenneth: Buybacks, Exit Bonds, and the Optimality of Debt and Liquidity Relief. International Economic Review 1989

Sachs Jeffrey: The Debt Overhang of Developing Countries. In: Findlay et al: Debt, Growth and Stabilization. Oxford 1988

Savvides Andreas: Investment Slowdown in Developing Countries during the 1980s: Debt Overhang or Foreign Capital Inflows? Kyklos 1992

Warner Andrew: Did the Debt Crisis cause the Investment Crisis? Quarterly Journal of Economics 1992 